\title{
Author Correction: Inducible overexpression of Ideal Plant Architecture1 improves both yield and disease resistance in rice
}

Mingming Liu, Zhenying Shi Di , Xiaohan Zhang, Mingxuan Wang, Lin Zhang, Kezhi Zheng, Jiyun Liu, Xingming Hu, Cuiru Di, Qian Qian, Zuhua He (D) and Dong-Lei Yang (D)

Correction to: Nature Plants https://doi.org/10.1038/s41477-019-0383-2, published online 18 March 2019.

In the Supplementary Information file originally published with this Article, the authors mistakenly omitted accompanying legends for Supplementary Figures 1-15; this has now been amended.

Published online: 16 May 2019

https://doi.org/10.1038/s41477-019-0447-3 